A Technique for Single-Pulse Spectrum and Pulse Width Measurements for an IR-FEL*

\author{
W. P. Leemans, J. A. Edighoffer, K.-J. Kim, S. Chattopadhyay \\ Lawrence Berkeley Laboratory \\ University of California \\ Berkeley, CA 94720 \\ and
}

H. A. Schwettman

W.W. Hansen Experimental Physics Laboratory

Stanford University, Stanford, CA 94305 - 4085 Presented at the Fourteenth International Free Electron Laser Conference, Kobe, Japan,
August 23-28, 1992 and to be published in the Proceedings.

* This work was supported in part by the Director, Office of Energy Research, Office of Basic Energy Sciences, Materials Sciences Division, of the U.S. Department of Energy under Contract No. DE-AC03-76SF00098, and by the Office of Naval Research, Contract No. N00014-91-C-0170.

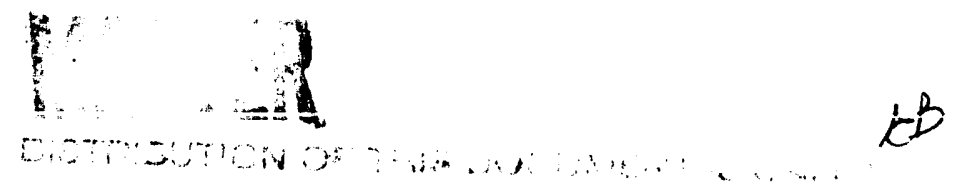




\title{
A Technique for Single-Pulse Spectrum and Pulse Width Measurements for an IR-FEL
}

\author{
W. P. Leemans, J. A. Edighoffer, K-J Kim, S. Chattopadhyay, \\ Lawrence Berkeley Laboratory, University of California, Berkeley CA 94720 \\ (510) 486 - 7788 \\ and \\ H. A. Schwettman \\ W.W Hansen Experimental Physics Laboratory \\ Stanford University, Stanford, CA 94305 - 4085, USA \\ (415) 723 - 0305
}

\begin{abstract}
A diagnostic system has been designed to measure the spectrum and pulse width of infrared FEL pulses. Use of an image dissector [1] and a single element high speed detector with integrating sphere, allows one to obtain spectral or temporal information for each individual micro-pulse inside the pulse train. The spectral diagnostic consisis of a mode matching telescope, a high resolution spectrometer, an imaging telescope and an image dissector system. The imaging telescope between the spectrograph and the image dissector provides for variable magnification (and hence resolution) of the spectrum onto the image dissector. The pulse width measurement uses single pulse autocorrelation through non-collinear optical mixing in a frequency doubling crystal [2], an imaging telescope and again an image dissector system. Here, the imaging telescope relays the image of the region in the nonlinear crystal in which second harmonic light was generated onto the image dissector. The design and implementation of these diagnostics for the $1-11 \mu \mathrm{m}$ on the IR-FEL at Stanford University are discussed.
\end{abstract}

This work was supported in part by the Director, Office of Energy Research, Office of Basic Energy Sciences, Material Sciences Division, of the U. S. Department of Energy under contract No. DE-AC0376SF00098, and by the Office of Naval Research, Contract No. N00014-91-C-0170. 


\section{Introduction}

The diagnostic system described in this paper was developed for the Stanford FEL, with applicability to the LBL Chemical Dynamics Research Laboratory (CDRL) IR-FEL. The Stanford FEL which has been in operation since 1983 [3], operates between 1.5 and $11 \mu \mathrm{m}$. The CDRL-IR-FEL will operate between 3 and $50 \mu \mathrm{m}$ [4]. The Stanford FEL is operated by a quasiCW superconducting linac, with an electron microbunch every 84 ns within a 5-10 ms macropulse. Table 1 shows the Stanford FEL operating parameters and the LBL CDRL-IR-FEL design parameters.

\begin{tabular}{llc} 
Wavelength $(\mu \mathrm{m})$ & Stanford FEL & $\begin{array}{c}\text { CDRL IR-FEL } \\
\text { (design) }\end{array}$ \\
Micropulse Energy $(\mu \mathrm{J})$ & $1.5-11$ & $3-50$ \\
Micropulse Rep Rate(MHz) & 12 & 100 \\
Macropulse Ave Power(W) & 12 & 6 \\
Duty Factor & $10 \%$ & 600 \\
Micropulse length(ps) & $1-3$ & $100 \%$ \\
$\begin{array}{l}\text { Transform Limited } \\
\text { Bandwidth @ 4 microns(\%) }\end{array}$ & $0.4-1.2$ & 30 \\
Wavelength Stability & $10^{-4}$ & 0.04 \\
\hline
\end{tabular}

Table 1: operating parameters of the Stanford FEL and design parameters for the proposed IRFEl at Lawrence Berkeley Laboratory.

The diagnostic techniques described here are capable of measuring the complete spectrum and the temporal pulse structure on each optical micropulse inside the macropulse. This detailed information is useful for both the laser operator and the user. Having the spectrum and pulse structure on a fast time scale allows the operator to increase the laser performance 
throughout the macropulse using fast feedback or feed forward stabilization. The user obtains information on the actual optical pulses being used in the experiment rather than a macropulse average. Indeed, the macropulse average spectrum will often be broader than the instantaneous spectrun due to fluctuations and sidebands.

The key concept behind obtaining spectral and temporal information for each individual micropulse inside the macropulse is the use of an image dissector [1]. In the image dissector an image enters a misaligned optical cavity. The optical cavity is misaligned so that on every round-trip a small slice of the image escapes the cavity. A detector looking at the output of the cavity will then record the image as being made up of consecutive slices separated in time by the cavity round-trip time.

The optical spectrum is obtained by dispersing the laser pulse in an optical spectrometer. The temporal pulse structure is measured using non-collinear second harmonic generation in a frequency doubling crystal. The transverse dimension of the region in which second harmonic generation occurred is a direct measure of the pulse width. In the case of the spectral diagnostic the image entering the dissector is the output spectrum from the spectrometer and in the case of the temporal diagnostic it is the region in which the second harmonic is generated. An other, much more expensive approach would be to buy a detector array operating at $12 \mathrm{MHz}$.

\section{Overall Layout}

The overall layout of the diagnostic system is shown in Figure 1. A fraction of the main FEL beam, heading towards the users, is split off using a beamsplitter and directed towards the spectrometer and autocorrelator set-up. The diagnostics share two HeNe laser beams $(0.6328 \mu \mathrm{m}$ and $3.39 \mu \mathrm{m}$ ) which are collinear with the FEL beam, for internal alignment and calibration purposes. Along the beam path several strategically placed kinematic mounts allow insertion of a quadrant detector, capable of measuring the positions of all three beams to about $20 \mu \mathrm{m}$. The FEL beam is mode matched into the spectrometer using a telescope, consisting of all metal 
mirrors, with variable magnification. The output of the spectrometer is imaged onto the input plane of an image dissector using again an all metal telescope with variable magnification. The output of the autocorrelator is also imaged onto the image dissector. Finally, the image dissector output is collected and focused onto a single element detector with a third all metal telescope.

\section{Detailed Description}

The spectrum is obtained using a 1 meter focal length f/10 spectrometer (SPEX 1000). The spectrometer covers the wavelength range from 3 to $11 \mu \mathrm{m}$ with two gratings, blazed at 4 and $7 \mu \mathrm{m}$, and having a groove density of 300 and 150 lines/mm respectively. The spectral coverage is $v$ riable from $0.2 \%$ to $20 \%$ by changing the magnification between the spectrometer exit and the image dissector, with the spectral resolution varying between $10^{-4}$ to $10^{-2}$.

The image dissector has two gold coated square mirrors (each with one edge beveled at $30^{\circ}$ and no chamfer) and a gold coated spherical mirror with a $22 \mathrm{~cm}$ radius of curvature. The image, roughly $1 \mathrm{~cm}$ long, enters the dissector over the sharp edge (horizontal) of the first (square) mirror and is imaged with the spherical mirror onto the third (square) mirror (Figure 3). A small slice of the image exits the dissector on the sharp edge (vertical) of the third mirror. The part of the image which did make it onto the third mirror is reimaged onto the first mirror with the same spherical mirror. To obtain the required resolution the image is divided into roughly 20 bins. To assure that the cavity is empty before the arrival of the next microbunch ( $84 \mathrm{~ns}$ later) we have chosen a cavity round-trip time of 2.9 ns resulting in a maximum temporal separation between first and last bin of approximately $60 \mathrm{~ns}$.

The output of the image dissector is demagnified to a $0.3 \mathrm{~mm} \times 1.5 \mathrm{~mm}$ size irnage onto a high speed detector. The detector contain s a high speed $\mathrm{HgCdTe}$ element $(100 \mu \mathrm{m} \times 100 \mu \mathrm{m})$ cooled at liquid nitrogen temperature and sensitive between 3 and $13 \mu \mathrm{m}$. The element is mounted inside a gold coated integrating sphere with a diameter of $7 \mathrm{~mm}$ and a $2 \mathrm{~mm}$ diameter entrance aperture. The photon half-life in the integrating sphere is about $200 \mathrm{ps,} \mathrm{well} \mathrm{below} \mathrm{the}$ cavity round-trip time. The integrating sphere is very inefficient, collecting on the detector 
element only about 1 part in 1200 of the light entering the sphere, but guarantees that the collection efficiency is independent of position or bin within the collected image.

The detector and pre-amplifier have a combined bandwidth of $600 \mathrm{MHz}$. The detector output is monitored on a digital oscilloscope which has a sampling rate of 1 gigasample/second and a $250 \mathrm{MHz}$ analog bandwidth. The measured system rise time is $800 \mathrm{ps}$. The overall bandwidth is large enough to completely reconstruct the original analog signal, filtered to 250 $\mathrm{MHz}$ (1.4 ns rise time), including the peaks and peak timing of each subinterval signal. Though feedback stabilization does not require complete signal reconstruction, it is required for displaying either the spectrum or autocorrelation measurement to the user or operator.

The detector has a measured current responsivity of $2.0 \mathrm{~A} / \mathrm{W}$ at $4 \mu \mathrm{m}$, a quantum efficiency of $60 \%$, and a theoretical noise equivalent power (NEP) of $0.032 \mathrm{nW} / \sqrt{\mathrm{Hz}}$. The preamplifier saturates at $1 \mathrm{~V}$ and has a $10 \mathrm{mV}$ noise floor, limiting the maximum signal-to-noise ratio $\mathrm{S} / \mathrm{N}$ to 100 . The overall measured voltage responsivity of the system, including spectrometer efficiency, mirror and integrating sphere losses, is $R V=7.4 \mathrm{~V} / \mu \mathrm{J}$.

We have obtained a 4 - 5 bin spectrum for each individual micropulse during the September - October FEL run at Stanford University. The peak signal level was around $250 \mathrm{mV}$ for the central spectral bin. During the next scheduled run we will increase the spectral resolution to 20 bins. If we assume that the FEL delivers $1 \mu \mathrm{J}$ to the user and that the image dissector causes a factor of 2 loss to the $20^{\text {th }}$ spectral bin, we expect $S / N=40$ and 20 for the first and last bin respectively. For greater accuracy, at the expense of temporal resolution, averaging over micropulses can be done.

The single pulse autocorrelator will use $\mathrm{AgGaS}_{2}$ or a $\mathrm{AgGaSe}$ crystal with about 1 to $5 \%$ efficiency over the 3 to $12.6 \mu \mathrm{m}$ range. The region in which the second harmonic is produced will be imaged onto the image dissector. The current system can not simultaneously measure both spectrum and pulse width. A future upgrade will allow combining the autocorrelator and spectrometer output at the entrance to the image dissector with a polarization sensitive beam splitter. Like-wise, the signals are split after the image dissector with another 
polarization sensitive beam splitter, sending the autocorrelation signal to a second fast detector (not shown in Figures 1 or 2.)

\section{Conclusion}

The spectral diagnostic system has been tested during the September - October 1992 experimental run at Stanford University. Figures 5 and 6 show pictures of the set-up before completion of the image dissector.

A micropulse spectrum with 4 - 5 spectral bins has been obtained. In the next experimental run, scheduled for January 1993, we will concentrate on increasing the spectral resolution to 20 bins. The measured sensitivity of the detector with integrating sphere is in agreement with the estimates. The software to correct for the image dissector attenuation and to provide feedback signals and user displays has yet to be written.

The autocorrelator is expected to be built and tested by the beginning of 1993 .

\section{References}

[1] H. A. Baldis, N. H. Burnett, and M. C. Richardson, Rev. Sci. Instrum. 48, 173 (1977).

[2] S. A. Arakelian, R. N. Gyuzalian, and S. B. Sogomonian, Optics Comm. 44, 67 (1982).

[3] J. A. Edighoffer et. al., Phys. Rev. Lett. 52, 5 (1984).

[4] "An Infrared Free-Electron Laser for the Chemical Dynamics Research Laboratory", Lawrence Berkeley Laboratory, Berkeley, CA., PUB-5335 (1992). 


\section{Figure Captions}

Figure 1: Table layout of the LBL/Stanford FEL diagnostics. The spectral and temporal diagnostic use $4 \%$ of the main FEL beam.

Figure 2: Spectrometer Systernconsisting of a polarization rotating periscope, an input mode matching telescope, two alignment HeNe lasers, quadrant detector kinematic mounts, 1-meter spectiometer, image magnifying telescope, image dissector, light gathering telescope and fast detector.

Figure 3: The lowest row on mirror 2 is the image of the entrance slit after $1 / 2$ round-trip. The next lowest row is the image of the entrance slit after 1 1/2 round trips minus the piece scraped off on the first pass and so on. The image spills out in bins separated in round-trip times, progressing from the right side of the entrance slit image to the left.

Figure 4: Single pulse autocorrelator based on non-collinear second harmonic generation. The beam is split into two equal beams with the same path length to the crystal center. The two beams cross at an angle in the crystal. The length of the region in which second harmonic light is generated is a direct measure of the optical pulse width.

Figure 5: A picture of the input side of tise spectrometer system showing one of the alignment lasers.

Figure 6: A picture of the output side of t.e spectrometer system. The large mirror and two small mirror mounts (lower middle portion of the picture) are part of the image dissector. 


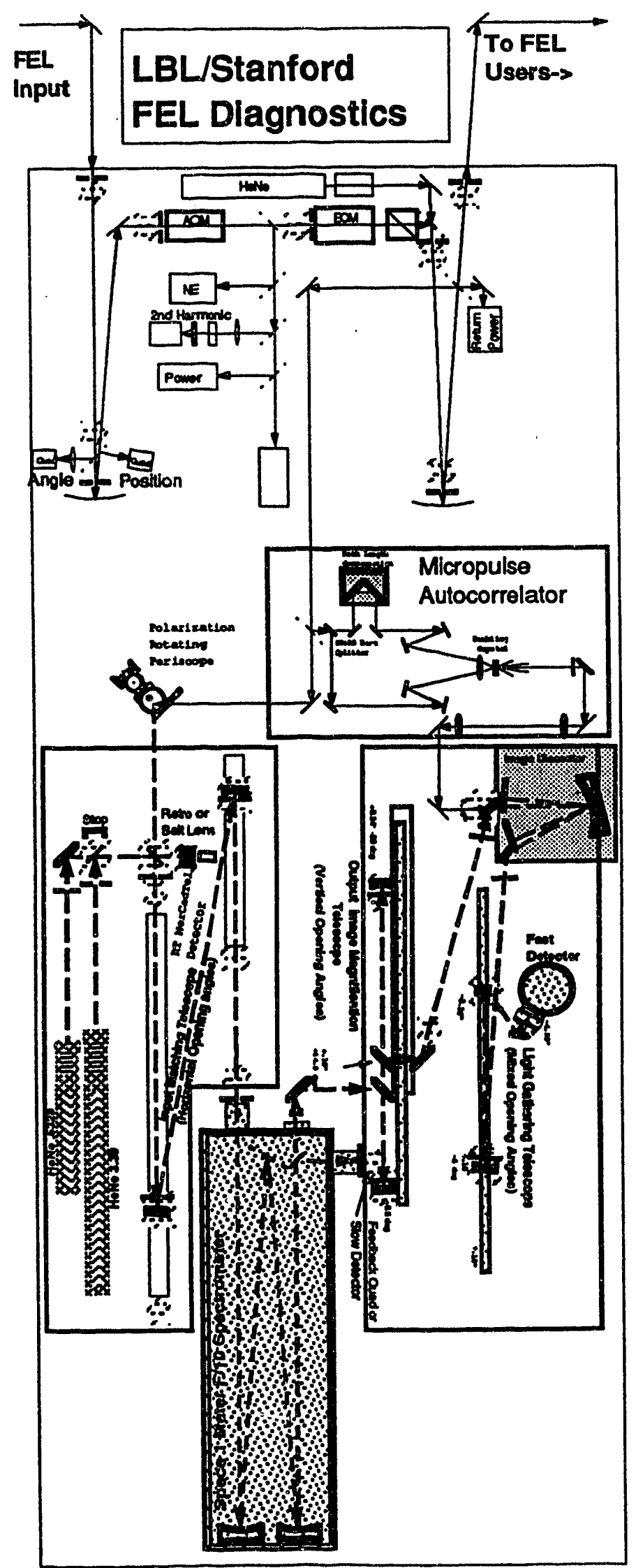




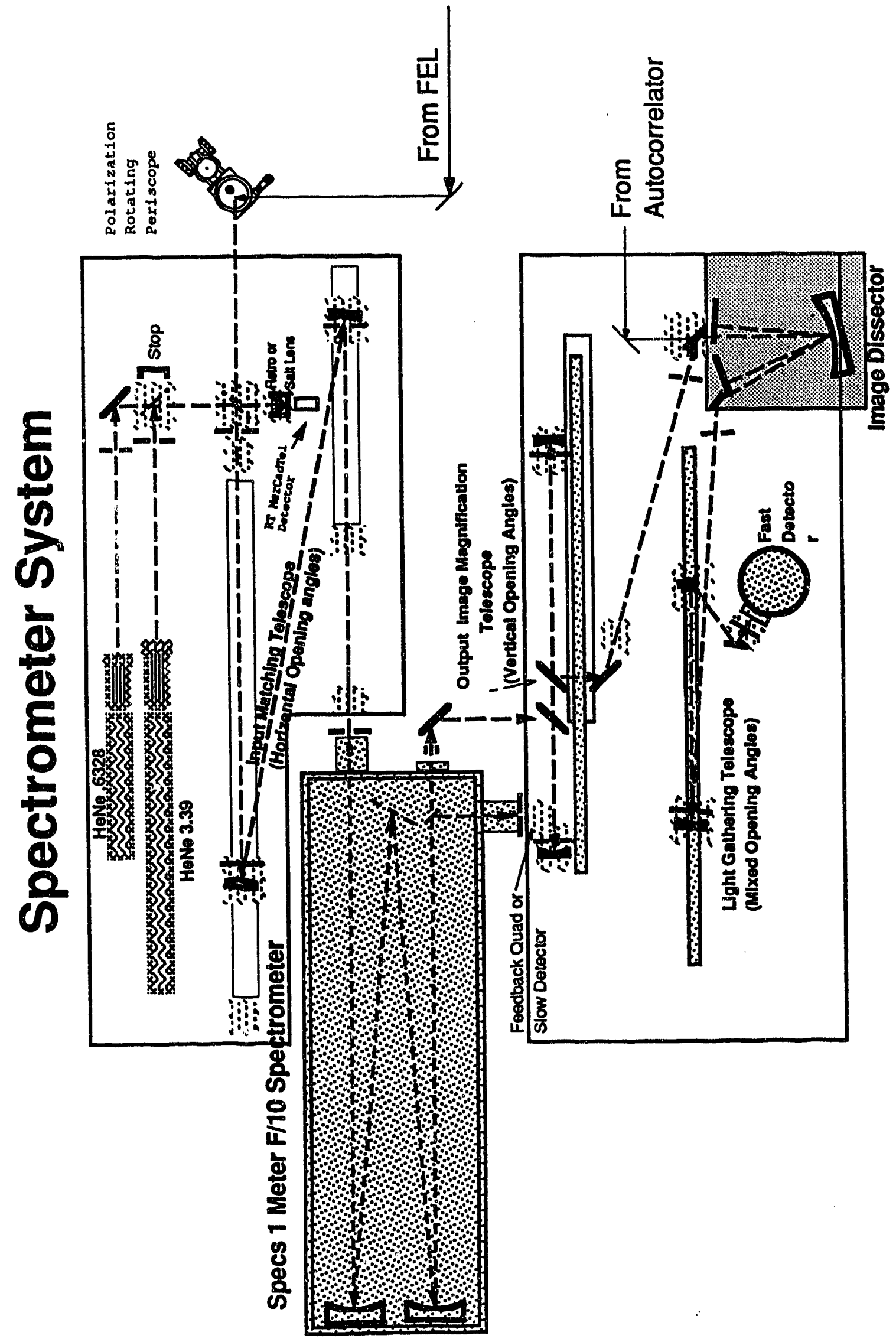




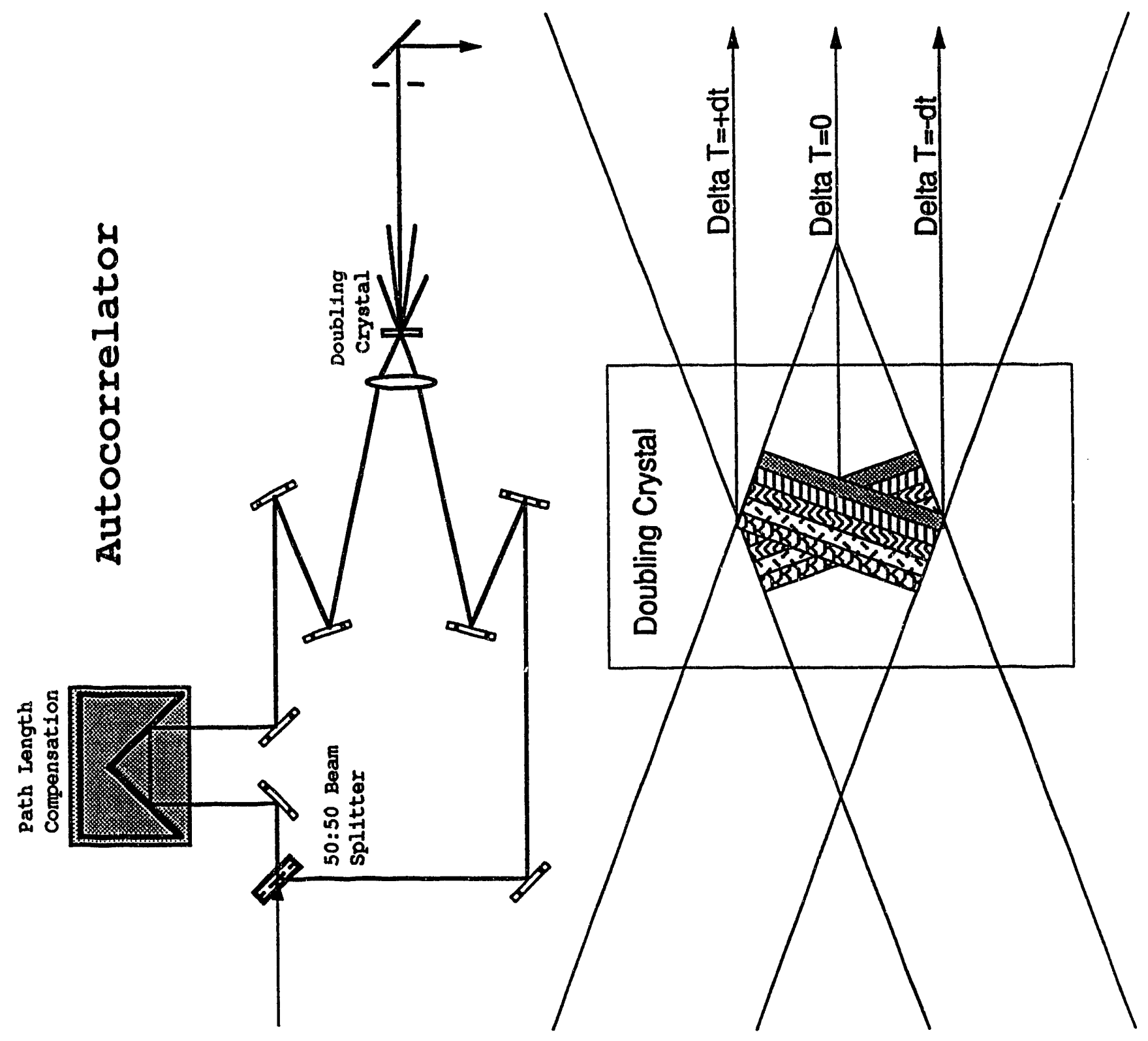




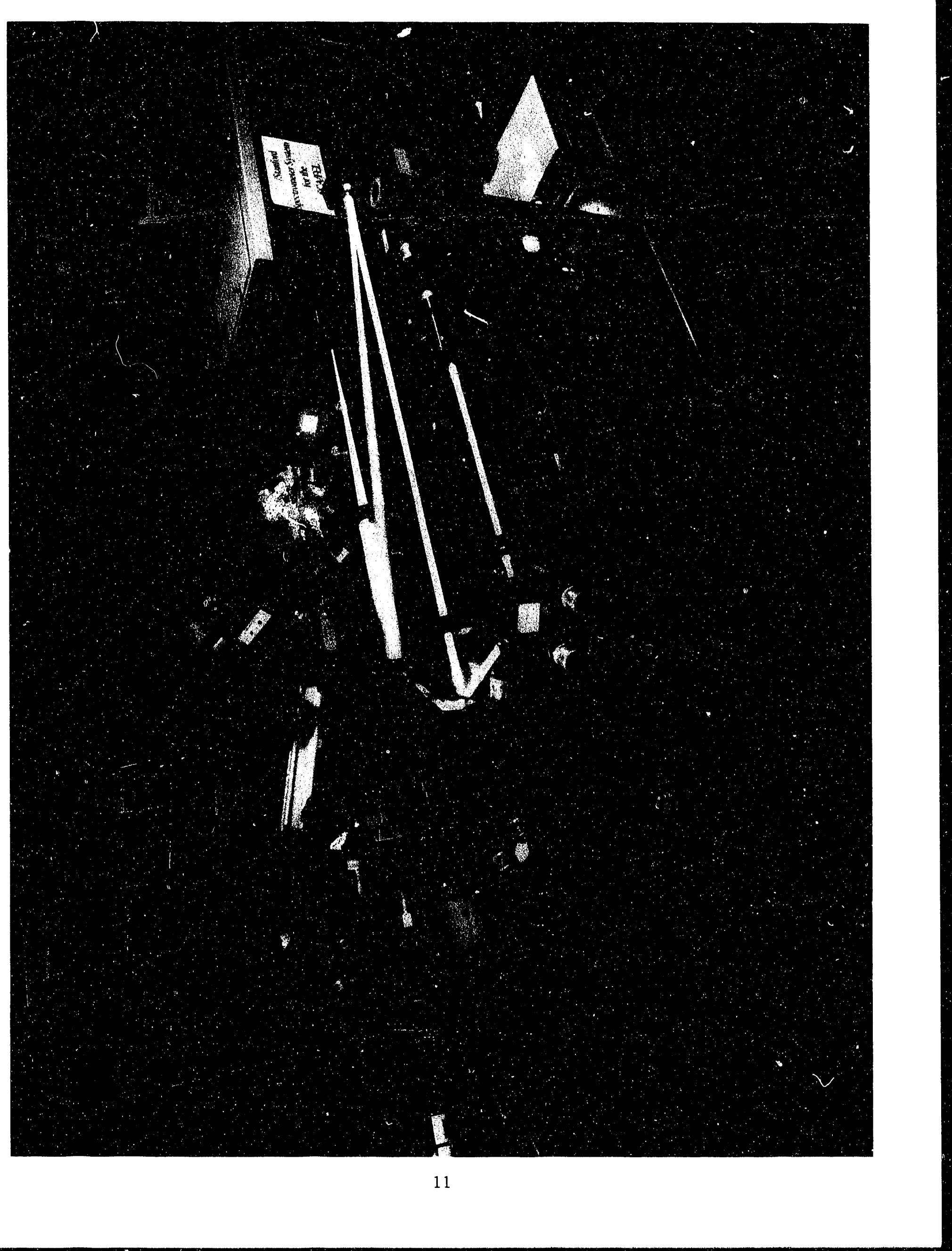




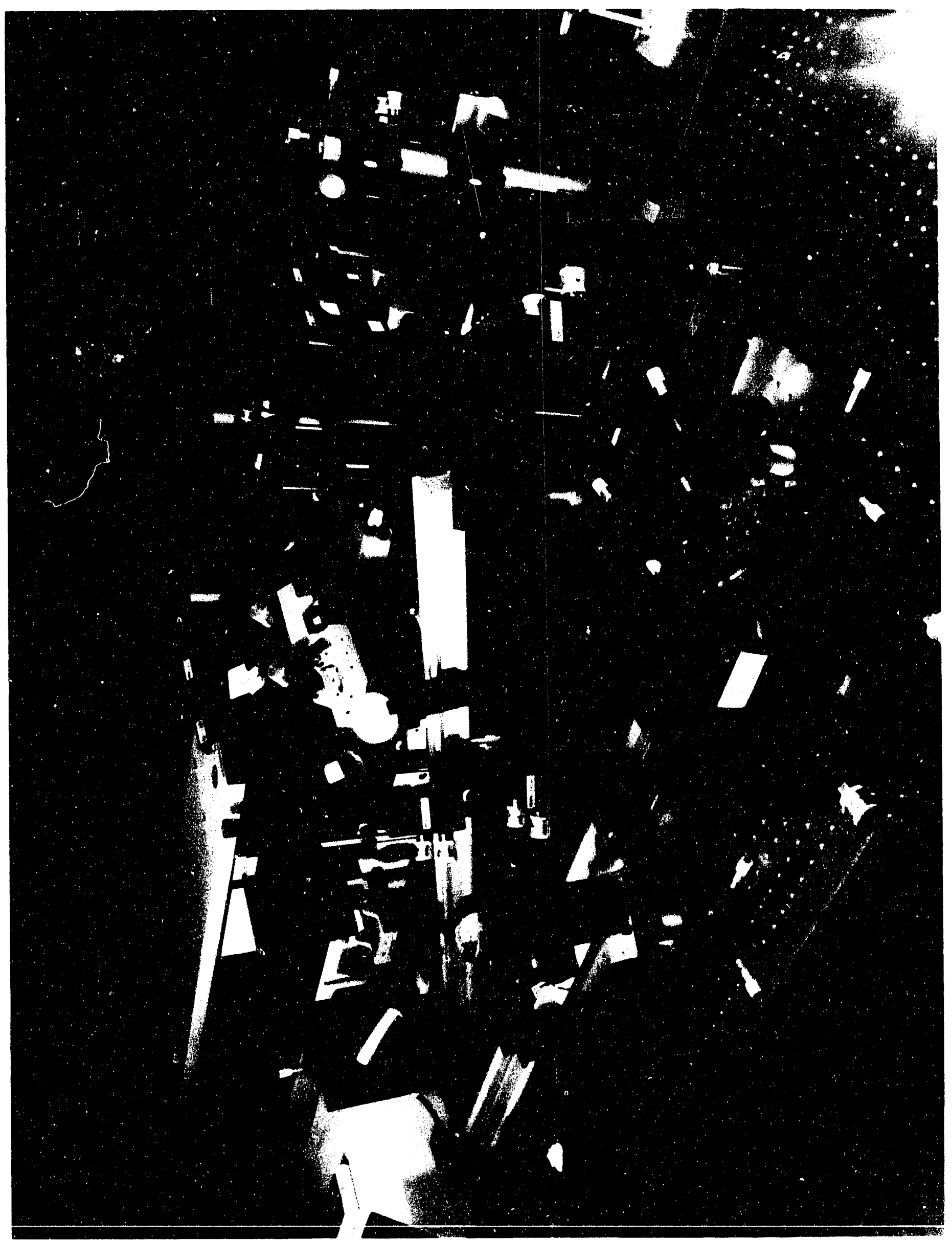




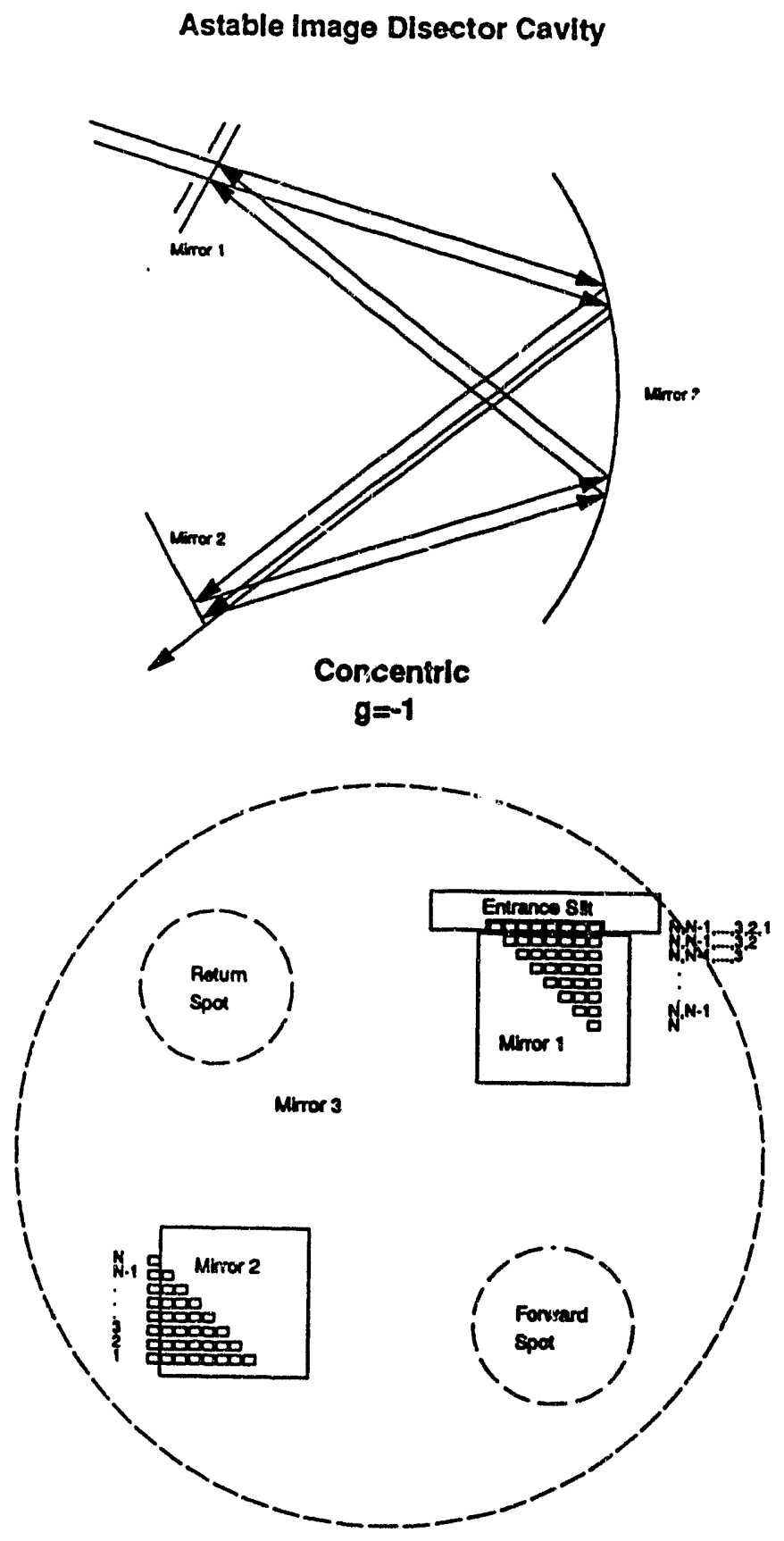

Image Disector BInning 

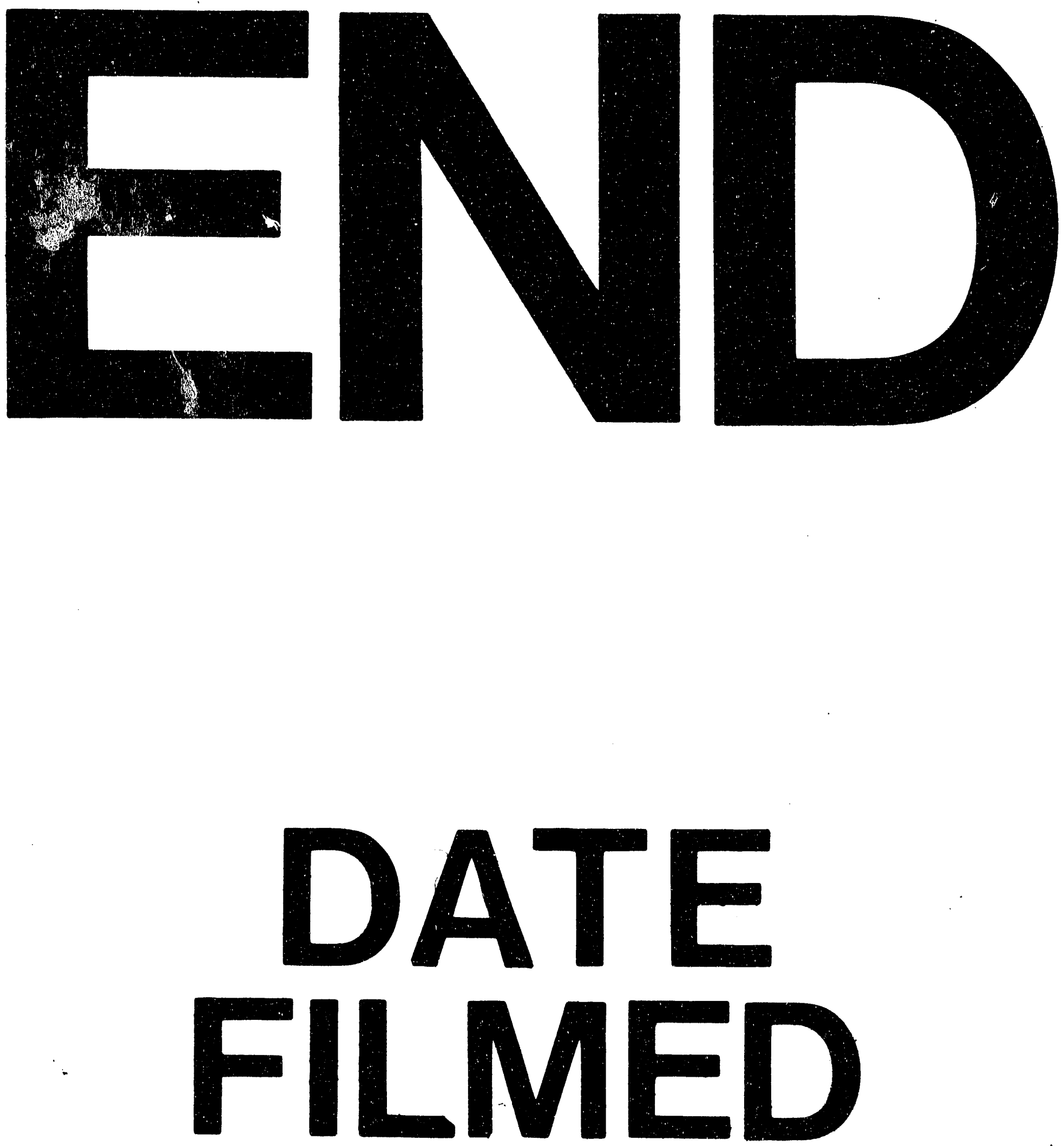

1

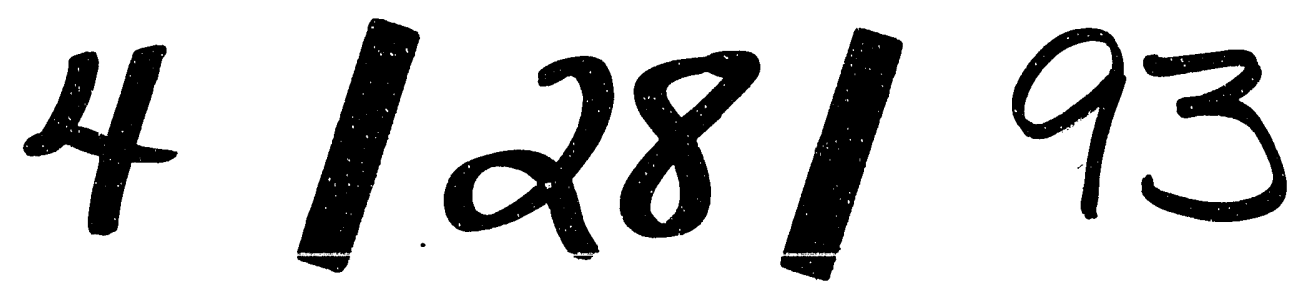


